Note

\title{
One-pot synthesis of nickel porphyrins with 2-pyridine-acetyl substituents: the unexpected Sonogashira cross-coupling products
}

\author{
Ching-Yao Lin ${ }^{\mathrm{a}, *}$, Li-Chieh Chuang ${ }^{\mathrm{a}}$, Gene-Hsiang Lee ${ }^{\mathrm{b}}$, Shie-Ming Peng ${ }^{\mathrm{b}}$ \\ a Department of Applied Chemistry, National Chi Nan University, 1, University Road, Puli, Nantou Hsien 545, Taiwan, ROC \\ b Department of Chemistry, National Taiwan University 1, Sec. 4, Roosevelt Road, Taipei 106, Taiwan, ROC
}

Received 16 January 2004; accepted 9 September 2004

Available online 7 October 2004

\begin{abstract}
Nickel porphyrins with 2-pyridine-acetyl substituents were synthesized in one step by the Sonogashira cross-coupling method. The structures of the products were determined by elemental analysis, ${ }^{1} \mathrm{H}$ NMR, UV-Vis, and X-ray spectroscopic techniques. It is suggested that cross-coupling bromonated nickel porphyrins with 2-pyridine-ethyne first yielded nickel porphyrins with 2-pyridine-ethynyl substituents, followed by in situ hydrolysis to the final products, nickel porphyrins with 2-pyridine-acetyl substituents. (c) 2004 Elsevier B.V. All rights reserved.
\end{abstract}

Keywords: 2-Acetylpyridine; Porphyrin; Cross-coupling; Hydrolysis

\section{Introduction}

2-Acetylpyridine is an important functional group found in many drugs [1], and it also has been widely used as the starting material for supramolecular systems [2], anti-tumor complexes [3], catalysis [4] and helicity study [5]. On the other hand, porphyrins have been extensively studied for multichromophore arrays, catalysis, photodynamic therapy and sensors because of their versatile electronic properties [6]. Combining the two molecules into one substance should bring new aspects and broaden the interests to the research; yet, synthesis of porphyrins with 2-pyridine-acetyl groups has never been reported. This is probably due to the complexity of the synthetic processes. For example, Grignard reagents [7] or the Suzuki coupling method [8] can be employed to connect 2-pyridine-acetyl group to porphyrins; however, both methods require several steps of preparation and separation. We hereby report a con-

\footnotetext{
* Corresponding author. Tel.: +886 49 2910960x4152; fax: +886 49 2917956.

E-mail address: cyl@ncnu.edu.tw (C.-Y. Lin).
}

venient, one-step synthesis of nickel porphyrins with 2pyridine-acetyl substituents. Fig. 1 depicts the structures of these porphyrins.

\section{Results and discussion}

The synthesis of 2-pyridine-acetyl nickel porphyrins involves reacting bromonated biphenylporphinato nickel(II) $\left(\mathrm{NiBPPBr}\right.$ or $\left.\mathrm{NiBPPBr}{ }_{2}\right)$ with 2-pyridylethyne [9]
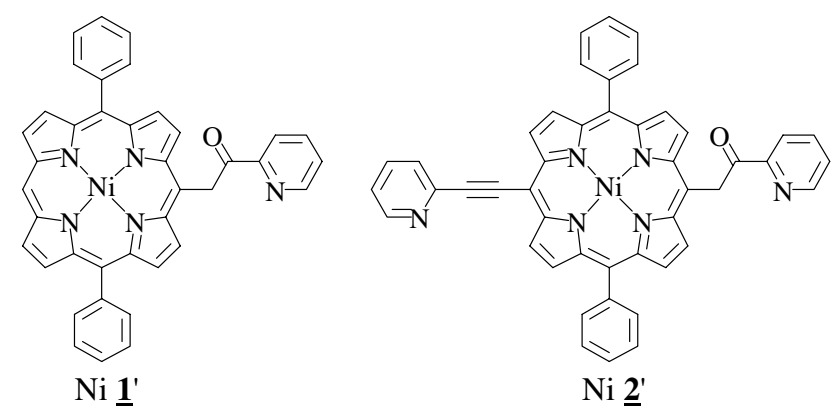

Fig. 1. Structure diagram of Ni porphyrins with 2-pyridine-acetyl substituents. 
according to the Sonogashira cross-coupling method [10]. This method has been widely used to synthesize porphyrins with ethyne-linked functional groups [1115]. Although porphyrins with 2-pyridine-ethynyl substituents were expected to be the products according to the method, we found that nickel porphyrins with 2pyridine-acetyl substituents were in fact the major products (Fig. 2). Two such reactions are shown in Fig. 2, and they afforded $55 \%$ of $\mathrm{Ni} \underline{\mathbf{1}}^{\prime}$ and $46 \%$ of $\mathrm{Ni} \underline{\mathbf{2}}^{\prime}$.

The NMR spectra of Ni $\underline{\mathbf{1}}^{\prime}$ and Ni $\underline{\mathbf{1}}$ are fairly similar to each other. For example, (1) the ${ }^{1} \mathrm{H}$ NMR peaks of $\mathrm{Ni} \underline{\mathbf{1}}^{\prime}$ differ from those of Ni $\underline{1}$ only by one singlet peak at $6.62 \mathrm{ppm}$ and (2) the other ${ }^{1} \mathrm{H}$ NMR peaks have similar chemical shifts and spin-spin splitting patterns (please see the characterization section for details). Therefore, the X-ray structure of Ni $\underline{\mathbf{1}}^{\prime}$ was obtained in order to more clearly identify these complexes (Fig. 3 , see also Tables 2 and 3). As shown in the figure, the acetyl group is indeed the group that connects the 2pyridine and the porphine core for $\mathrm{Ni} \underline{\underline{1}}^{\prime}$. Based on this, the ${ }^{1} \mathrm{H}$ NMR spectra of $\mathrm{Ni} \underline{\mathbf{1}}^{\prime}$ and $\mathrm{Ni} \underline{\mathbf{1}}$ were better understood. More importantly, the ${ }^{1} \mathrm{H}$ NMR peaks of Ni $\underline{\mathbf{2}}^{\prime}$ were properly assigned, namely to distinguish the ${ }^{1} \mathrm{H}$ NMR peaks of the 2-pyridine-acetyl group and those of the 2-pyridine-ethynyl substituent.

On the other hand, the UV-Vis absorptions of NiBPP, Ni $\underline{1}$ and Ni $\underline{\mathbf{1}}^{\prime}$ reveal different degrees of the interactions between the substituents and the porphine core (Fig. 4): Ni 1 has the most red-shifted absorptions owing to the better resonance interactions via the ethynyl bridge. In contrast, the acetyl group provides less effective $\pi$-interactions between the substituents and the porphine ring, resulting in the less red-shifted absorptions of Ni $\underline{\mathbf{1}}^{\prime}$.

To further our understanding to these unexpected products, the reactions were analyzed by systematically changing the experimental conditions. The results are compared in Table 1. As shown in the table, the complex

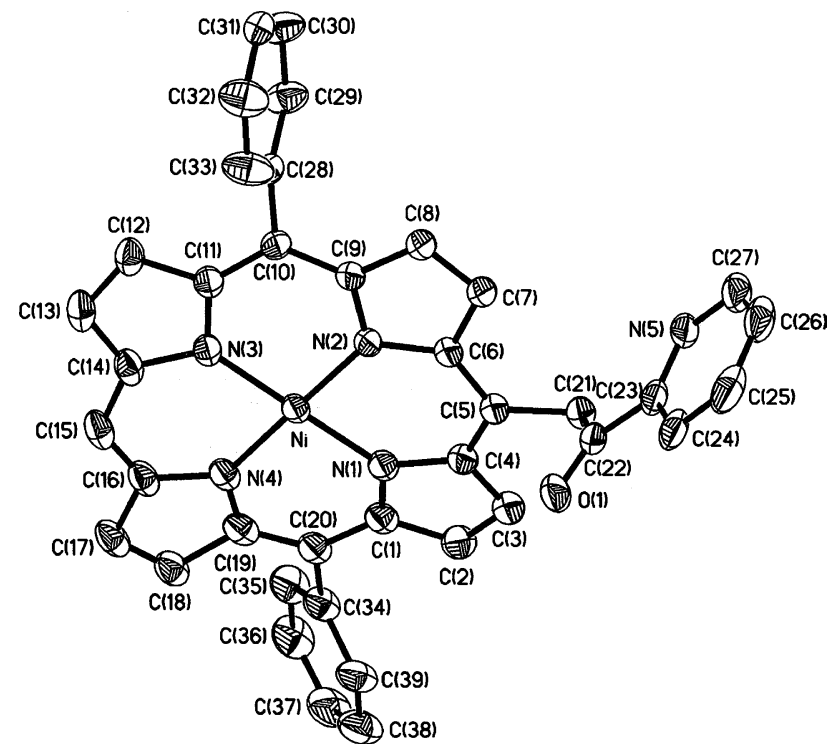

Fig. 3. Crystal structure of $\mathrm{Ni} \underline{\underline{\mathbf{1}}}^{\prime}$.

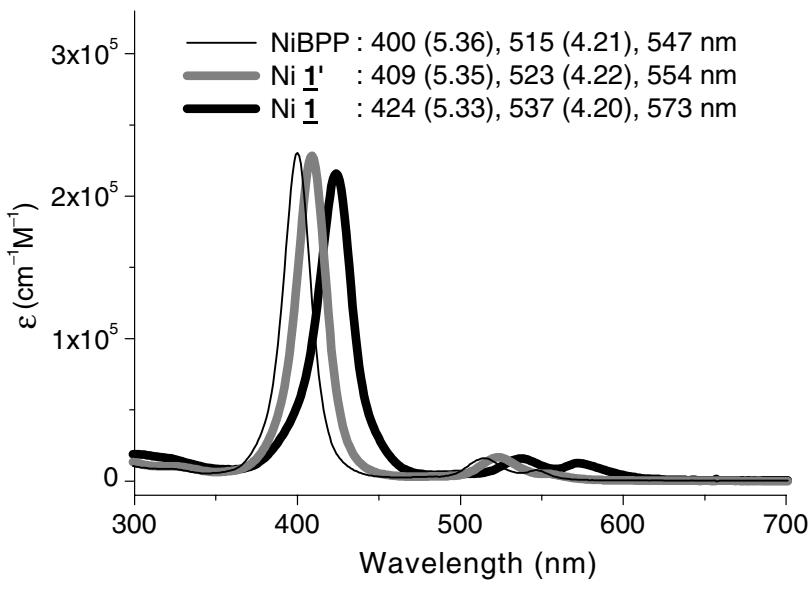

Fig. 4. The absorption spectra of NiBPP, Ni $\underline{\underline{1}}^{\prime}$ and $\mathrm{Ni} \underline{\mathbf{1}}$ in $\mathrm{CH}_{2} \mathrm{Cl}_{2}$ ( $\log \varepsilon$ shown in the parentheses).
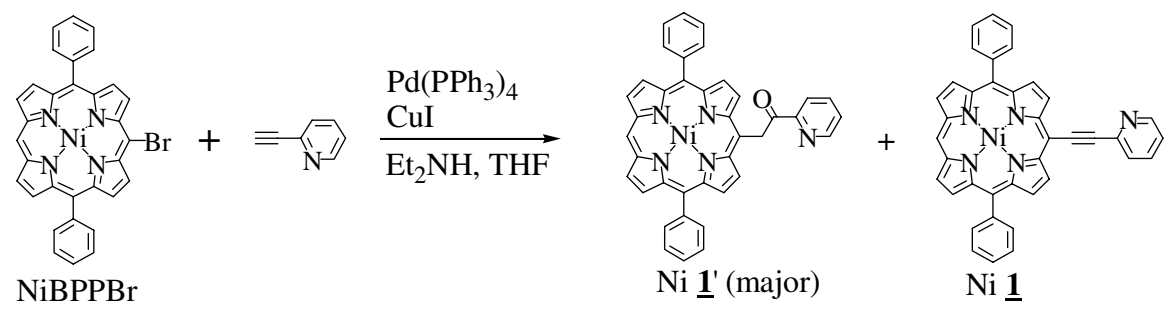

(a)
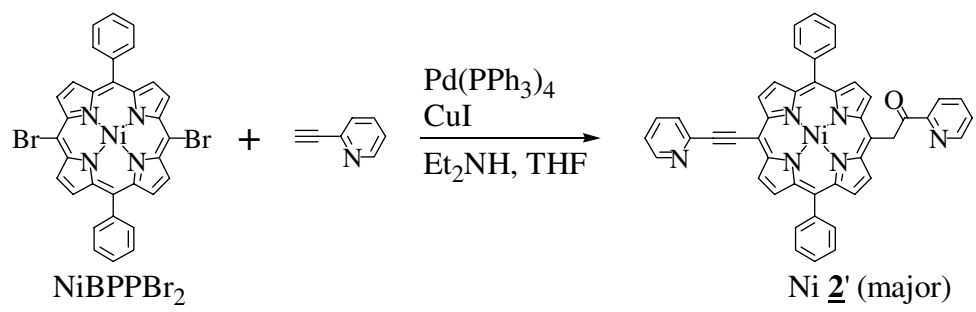

(b)

Fig. 2. Reacting bromonated NiBPP with 2-pyridylethyne. 
Table 1

Selected reaction conditions and yields

\begin{tabular}{|c|c|c|c|}
\hline Starting materials & Reaction conditions & Reaction time & Products \\
\hline $\mathrm{NiBPPBr} / 2 \mathrm{PyCCH}$ & $\mathrm{Pd}\left(\mathrm{PPh}_{3}\right)_{4} / \mathrm{CuI} / \mathrm{Et}_{2} \mathrm{NH} / \mathrm{THF}$ & $38 \mathrm{~h}^{\mathrm{a}}$ & $41 \% \mathrm{Ni} \underline{\mathbf{1}}^{\prime}+21 \% \mathrm{Ni} \underline{\mathbf{1}}$ \\
\hline $\mathrm{NiBPPBr} / 2 \mathrm{PyCCH}$ & $\mathrm{Pd}\left(\mathrm{PPh}_{3}\right)_{4} / \mathrm{CuI} / \mathrm{Et}_{2} \mathrm{NH} / \mathrm{THF}$ & 5 days & $55 \% \mathrm{Ni} \underline{\mathbf{1}}^{\prime}$ \\
\hline $\mathrm{Ni} \underline{1}$ & $\mathrm{Pd}\left(\mathrm{PPh}_{3}\right)_{4} / \mathrm{CuI} / \mathrm{Et}_{2} \mathrm{NH} / \mathrm{THF}^{\mathrm{b}}$ & 5 days & $55 \% \mathrm{Ni} \underline{\mathbf{1}}^{\prime}$ \\
\hline $\mathrm{NiBPPBr}_{2} / 2 \mathrm{PyCCH}$ & $\mathrm{Pd}\left(\mathrm{PPh}_{3}\right)_{4} / \mathrm{CuI} / \mathrm{Et}_{2} \mathrm{NH} / \mathrm{THF}$ & $43 \mathrm{~h}^{\mathrm{c}}$ & $46 \% \mathrm{Ni} \underline{\mathbf{2}}^{\prime}$ \\
\hline $\mathrm{ZnBPPBr} / 2 \mathrm{PyCCH}$ & $\mathrm{Pd}\left(\mathrm{PPh}_{3}\right)_{4} / \mathrm{CuI} / \mathrm{Et}_{2} \mathrm{NH} / \mathrm{THF}$ & 5 days & $<2 \% \operatorname{Zn} \underline{\mathbf{1}}^{\prime}+21 \% \operatorname{Zn} \underline{\mathbf{1}}$ \\
\hline $\mathrm{ZnBPPBr} / 2 \mathrm{PyCCH}$ & $\mathrm{Pd}\left(\mathrm{PPh}_{3}\right)_{4} / \mathrm{CuI} / \mathrm{Et}_{2} \mathrm{NH} / \mathrm{THF}+1$ drop $\mathrm{H}_{2} \mathrm{O}^{\mathrm{d}}$ & 5 days & $<5 \% \operatorname{Zn} \underline{\mathbf{1}}^{\prime}+21 \% \operatorname{Zn} \underline{\mathbf{1}}^{\mathrm{e}}$ \\
\hline
\end{tabular}

a NiBPPBr was not completely consumed at this point.

b None of the reagent alone converted Ni $\underline{1}$ to $\mathrm{Ni} \underline{\mathbf{1}}^{\prime}$.

c Prolonged reaction time decomposes the porphyrins, and multiple bands were observed in column.

d Adding one drop of water did not significantly affect the nickel porphyrin reactions.

e Prolonged reaction time decreased the overall yields of the porphyrins. The yields were higher if shortened the reaction time [18].

$\mathrm{Ni} 1 \mathrm{was}$ found to be one of the reaction products at $38 \mathrm{~h}$ in the cross-coupling reactions (Entry 1). However, this compound completely disappeared after prolonged reaction and the yield of $\mathrm{Ni} \underline{\mathbf{1}}^{\prime}$ increased from $41 \%$ to $55 \%$. More importantly, although very stable in THF, Ni $\underline{1}$ was converted to $\mathrm{Ni} \underline{\mathbf{1}}^{\prime}$ at $55 \%$ yield after standing in the cross-coupling environment for five days (Entry 3). These results suggest that (1) Ni $\underline{1}$ was the initial product of the cross-coupling reactions, and (2) Ni 1 was gradually converted to $\mathrm{Ni} \underline{1}^{\prime}$ in situ and (3) the conversion rate from Ni $\underline{1}$ to $\mathrm{Ni} \underline{\mathbf{1}}^{\prime}$ was $55 \%$ (see Tables 2 and 3 ).

Many acid-hydrolysis reactions of pyridylethyne have been well documented [16], therefore, it is logical to assume that $\mathrm{Ni} \underline{1}$ transforms to $\mathrm{Ni} \underline{\mathbf{1}}^{\prime}$ via the hydrolysis mechanism. Furthermore, the conversion rate of 2-pyridylethyne has been reported to be $60 \%$; this is quite close to the $\mathrm{Ni} \underline{\mathbf{1}}^{\prime}$ conversion rate $(55 \%$, from $\mathrm{Ni} \underline{\mathbf{1}})$. Note-worthily, converting $\mathrm{Ni} \underline{\mathbf{1}}$ to $\mathrm{Ni} \underline{\mathbf{1}}^{\prime}$ does not require the intro-

Table 2

Crystal data for Ni $\underline{\mathbf{1}}^{\prime}$

\begin{tabular}{ll}
\hline Crystallizes from $\mathrm{CH}_{2} \mathrm{Cl}_{2}$ /hexane as dark-red blocks \\
Formula & $\mathrm{C}_{39} \mathrm{H}_{25} \mathrm{~N}_{5} \mathrm{NiO}$ \\
Formula weight & 638.35 \\
Temperature $(\mathrm{K})$ & $150(1)$ \\
Wavelength $(\AA)$ & 0.71073 \\
Crystal system & Monoclinic \\
Space group & $C 2 / c$ \\
Unit cell dimensions & \\
$\quad a(\AA)$ & $25.4032(4)$ \\
$\quad b(\AA)$ & $13.1159(2)$ \\
$\quad c(\AA)$ & $17.8961(3)$ \\
Volume $\left(\AA^{3}\right)$ & $5846.85(16)$ \\
$Z$ & 8 \\
Density (calculated) $\left(\mathrm{mg} / \mathrm{m}^{3}\right)$ & 1.450 \\
Absorption coefficient $\left(\mathrm{mm}^{-1}\right)$ & 0.707 \\
$F(000)$ & 2640 \\
Crystal size (mm $\left.{ }^{3}\right)$ & $35 \times 30 \times 25$ \\
$\theta$ Range for data collection $\left({ }^{\circ} \mathrm{C}\right)$ & $1.64-27.50$ \\
Reflections collected & 49,721 \\
Independent reflections & $6722\left(R_{\text {int }}=0.0827\right)$ \\
Goodness-to-fit on $F^{2}$ & 1.188 \\
Final $R$ indices $[I>2 \sigma(I)]$ & $R_{1}=0.0749, w R_{2}=0.2001$ \\
$R$ indices (all data) & $R_{1}=0.1012, w R_{2}=0.2249$ \\
Extinction coefficient & $0.0126(9)$ \\
\hline
\end{tabular}

duction of acids. In addition, the source of water for hydrolysis in the reaction solutions could be from the crystallized porphyrins or the palladium catalyst [17]. We found that such trace amount of water was enough for the conversion, and adding extra water did not increase the yield of $\mathrm{Ni} \underline{1}^{\prime}$ nor shorten the reaction time.

We also examined the same reactions with different porphyrins: reacting 2-pyridylethyne with $\mathrm{NiBPPBr}_{2}$ yielded $46 \%$ of $\mathrm{Ni} \underline{\mathbf{2}}^{\prime}$ after $43 \mathrm{~h}$ of reaction (Table 1, Entry 4). In this case, prolonging reaction time decomposed the porphyrins and numerous bands were found during chromatographic separation. Also, the same phenomenon is far less effective for the zinc analogues, and the yields were less than those observed for the nickel complexes (Table 1, Entry 5). Interestingly, adding extra water seemed to increase the yield of $\mathrm{Zn} \underline{\mathbf{1}}^{\prime}$ (Entry 6). The difference between the nickel and the zinc porphyrin reactions is possibly due to the facts that: (1) zinc(II) is a $\mathrm{d}^{10}$ close-shell ion and nickel(II) is a $\mathrm{d}^{8}$ open-shell ion, and (2) zinc(II) porphyrins form the 5- or 6-coordinated complex fairly easily [19] while nickel(II) porphyrins are usually 4-coordinated unless dissolved in strongly ligating solvents, such as pyridine or piperidine [20]. These two factors are likely to affect the geometry, the energy and the electronic structure of these porphyrins, resulting in the difference in the reactivities.

In summary, we report a one-pot synthesis of nickel porphyrins with 2-pyridine-acetyl substituents by the Sonogashira cross-coupling method. It is suggested that porphyrins with 2-pyridine-ethynyl substituents were first generated, and were gradually converted to porphyrins with 2-pyridine-acetyl substituents via in situ hydrolysis.

\section{Experimental}

\subsection{Materials}

Air-sensitive solids were handled in an MBraun Unilab glove box. A vacuum line and standard Schlenk techniques were employed to process air-sensitive solu- 
Table 3

Selected bond lengths and bond angles of Ni $\underline{\mathbf{1}}^{\prime}$

\begin{tabular}{|c|c|c|c|c|c|}
\hline \multicolumn{6}{|c|}{ Bond lengths (̊̊) } \\
\hline $\mathrm{Ni}-\mathrm{N} 2$ & $1.932(3)$ & N5-C23 & $1.341(6)$ & $\mathrm{C} 12-\mathrm{C} 13$ & $1.341(6)$ \\
\hline $\mathrm{Ni}-\mathrm{N} 4$ & $1.940(3)$ & $\mathrm{C} 1-\mathrm{C} 20$ & $1.406(5)$ & $\mathrm{C} 13-\mathrm{C} 14$ & $1.414(7)$ \\
\hline $\mathrm{Ni}-\mathrm{N} 1$ & $1.952(3)$ & $\mathrm{C} 1-\mathrm{C} 2$ & $1.434(6)$ & $\mathrm{C} 14-\mathrm{C} 15$ & $1.384(6)$ \\
\hline $\mathrm{Ni}-\mathrm{N} 3$ & $1.958(3)$ & $\mathrm{C} 2-\mathrm{C} 3$ & $1.334(6)$ & $\mathrm{C} 15-\mathrm{C} 16$ & $1.359(7)$ \\
\hline $\mathrm{O} 1-\mathrm{C} 22$ & $1.212(5)$ & $\mathrm{C} 3-\mathrm{C} 4$ & $1.440(6)$ & $\mathrm{C} 16-\mathrm{C} 17$ & $1.437(6)$ \\
\hline $\mathrm{N} 1-\mathrm{C} 1$ & $1.370(5)$ & $\mathrm{C} 4-\mathrm{C} 5$ & $1.387(6)$ & $\mathrm{C} 17-\mathrm{C} 18$ & $1.340(8)$ \\
\hline $\mathrm{N} 1-\mathrm{C} 4$ & $1.392(5)$ & C5-C6 & $1.391(6)$ & $\mathrm{C} 18-\mathrm{C} 19$ & $1.442(5)$ \\
\hline $\mathrm{N} 2-\mathrm{C} 6$ & $1.378(5)$ & $\mathrm{C} 5-\mathrm{C} 21$ & $1.513(5)$ & $\mathrm{C} 19-\mathrm{C} 20$ & $1.390(6)$ \\
\hline $\mathrm{N} 2-\mathrm{C} 9$ & $1.387(5)$ & $\mathrm{C} 6-\mathrm{C} 7$ & $1.433(6)$ & $\mathrm{C} 21-\mathrm{C} 22$ & $1.517(7)$ \\
\hline N3-C11 & $1.376(5)$ & $\mathrm{C} 7-\mathrm{C} 8$ & $1.347(7)$ & $\mathrm{C} 22-\mathrm{C} 23$ & $1.495(5)$ \\
\hline N3-C14 & $1.378(5)$ & $\mathrm{C} 8-\mathrm{C} 9$ & $1.426(6)$ & $\mathrm{C} 23-\mathrm{C} 24$ & $1.387(8)$ \\
\hline N4-C19 & $1.374(6)$ & $\mathrm{C} 9-\mathrm{C} 10$ & $1.384(6)$ & $\mathrm{C} 24-\mathrm{C} 25$ & $1.388(7)$ \\
\hline $\mathrm{N} 4-\mathrm{C} 16$ & $1.400(5)$ & $\mathrm{C} 10-\mathrm{C} 11$ & $1.376(5)$ & $\mathrm{C} 25-\mathrm{C} 26$ & $1.390(11)$ \\
\hline N5-C27 & $1.334(6)$ & $\mathrm{C} 11-\mathrm{C} 12$ & $1.439(6)$ & $\mathrm{C} 26-\mathrm{C} 27$ & $1.375(11)$ \\
\hline \multicolumn{6}{|l|}{ Bond angle $\left({ }^{\circ}\right)$} \\
\hline $\mathrm{N} 2-\mathrm{Ni}-\mathrm{N} 4$ & $179.22(15)$ & $\mathrm{C} 2-\mathrm{C} 3-\mathrm{C} 4$ & $107.0(4)$ & $\mathrm{C} 15-\mathrm{C} 14-\mathrm{C} 13$ & $123.8(4)$ \\
\hline $\mathrm{N} 2-\mathrm{Ni}-\mathrm{N} 1$ & $90.43(13)$ & $\mathrm{C} 5-\mathrm{C} 4-\mathrm{N} 1$ & $125.1(4)$ & $\mathrm{C} 16-\mathrm{C} 15-\mathrm{C} 14$ & $124.2(4)$ \\
\hline $\mathrm{N} 4-\mathrm{Ni}-\mathrm{N} 1$ & $88.94(14)$ & $\mathrm{C} 5-\mathrm{C} 4-\mathrm{C} 3$ & $124.3(4)$ & $\mathrm{C} 15-\mathrm{C} 16-\mathrm{N} 4$ & $125.7(4)$ \\
\hline $\mathrm{N} 2-\mathrm{Ni}-\mathrm{N} 3$ & $89.42(13)$ & $\mathrm{N} 1-\mathrm{C} 4-\mathrm{C} 3$ & $110.5(4)$ & $\mathrm{C} 15-\mathrm{C} 16-\mathrm{C} 17$ & $124.3(4)$ \\
\hline $\mathrm{N} 4-\mathrm{Ni}-\mathrm{N} 3$ & $91.21(14)$ & $\mathrm{C} 4-\mathrm{C} 5-\mathrm{C} 6$ & $121.8(4)$ & $\mathrm{N} 4-\mathrm{C} 16-\mathrm{C} 17$ & $109.9(4)$ \\
\hline $\mathrm{N} 1-\mathrm{Ni}-\mathrm{N} 3$ & $179.18(12)$ & $\mathrm{C} 4-\mathrm{C} 5-\mathrm{C} 21$ & $121.0(4)$ & $\mathrm{C} 18-\mathrm{C} 17-\mathrm{C} 16$ & 107.7(4) \\
\hline $\mathrm{C} 1-\mathrm{N} 1-\mathrm{C} 4$ & $103.8(3)$ & C6-C5-C21 & $117.3(3)$ & $\mathrm{C} 17-\mathrm{C} 18-\mathrm{C} 19$ & $106.8(4)$ \\
\hline $\mathrm{C} 1-\mathrm{N} 1-\mathrm{Ni}$ & $128.4(3)$ & $\mathrm{N} 2-\mathrm{C} 6-\mathrm{C} 5$ & $126.5(4)$ & $\mathrm{N} 4-\mathrm{C} 19-\mathrm{C} 20$ & $125.6(3)$ \\
\hline $\mathrm{C} 4-\mathrm{N} 1-\mathrm{Ni}$ & 127.7(3) & $\mathrm{N} 2-\mathrm{C} 6-\mathrm{C} 7$ & $110.7(4)$ & N4-C19-C18 & 111.1(4) \\
\hline $\mathrm{C} 6-\mathrm{N} 2-\mathrm{C} 9$ & $104.9(3)$ & $\mathrm{C} 5-\mathrm{C} 6-\mathrm{C} 7$ & $122.7(4)$ & $\mathrm{C} 20-\mathrm{C} 19-\mathrm{C} 18$ & $123.3(4)$ \\
\hline $\mathrm{C} 6-\mathrm{N} 2-\mathrm{Ni}$ & $127.4(3)$ & C8-C7-C6 & $106.7(4)$ & $\mathrm{C} 19-\mathrm{C} 20-\mathrm{C} 1$ & $120.6(4)$ \\
\hline C9-N2-Ni & $127.7(2)$ & $\mathrm{C} 7-\mathrm{C} 8-\mathrm{C} 9$ & $107.8(4)$ & $\mathrm{C} 5-\mathrm{C} 21-\mathrm{C} 22$ & $111.4(4)$ \\
\hline C11-N3-C14 & $104.3(3)$ & $\mathrm{C} 10-\mathrm{C} 9-\mathrm{N} 2$ & $126.5(3)$ & $\mathrm{O} 1-\mathrm{C} 22-\mathrm{C} 23$ & $119.9(4)$ \\
\hline C11-N3-Ni & $128.8(2)$ & C10-C9-C8 & $123.4(4)$ & $\mathrm{O} 1-\mathrm{C} 22-\mathrm{C} 21$ & $120.8(4)$ \\
\hline C14-N3-Ni & $126.9(3)$ & $\mathrm{N} 2-\mathrm{C} 9-\mathrm{C} 8$ & $110.0(3)$ & $\mathrm{C} 23-\mathrm{C} 22-\mathrm{C} 21$ & $119.3(4)$ \\
\hline C19-N4-C16 & $104.5(3)$ & C11-C10-C9 & 121.9(4) & N5-C23-C24 & $124.6(4)$ \\
\hline C19-N4-Ni & $129.4(3)$ & $\mathrm{C} 10-\mathrm{C} 11-\mathrm{N} 3$ & $125.0(4)$ & N5-C23-C22 & $117.0(5)$ \\
\hline C16-N4-Ni & $126.0(3)$ & $\mathrm{C} 10-\mathrm{C} 11-\mathrm{C} 12$ & $124.5(4)$ & $\mathrm{C} 24-\mathrm{C} 23-\mathrm{C} 22$ & $118.3(4)$ \\
\hline $\mathrm{C} 27-\mathrm{N} 5-\mathrm{C} 23$ & $116.4(6)$ & N3-C11-C12 & $110.4(3)$ & $\mathrm{C} 23-\mathrm{C} 24-\mathrm{C} 25$ & $118.2(6)$ \\
\hline $\mathrm{N} 1-\mathrm{C} 1-\mathrm{C} 20$ & $125.9(4)$ & $\mathrm{C} 13-\mathrm{C} 12-\mathrm{C} 11$ & $106.8(4)$ & $\mathrm{C} 24-\mathrm{C} 25-\mathrm{C} 26$ & $117.3(7)$ \\
\hline $\mathrm{N} 1-\mathrm{C} 1-\mathrm{C} 2$ & $111.6(3)$ & $\mathrm{C} 12-\mathrm{C} 13-\mathrm{C} 14$ & 107.1(4) & $\mathrm{C} 27-\mathrm{C} 26-\mathrm{C} 25$ & $120.4(5)$ \\
\hline $\mathrm{C} 20-\mathrm{C} 1-\mathrm{C} 2$ & $122.5(4)$ & N3-C14-C15 & $124.8(4)$ & N5-C27-C26 & $123.1(6)$ \\
\hline $\mathrm{C} 3-\mathrm{C} 2-\mathrm{C} 1$ & $106.9(4)$ & $\mathrm{N} 3-\mathrm{C} 14-\mathrm{C} 13$ & $111.4(4)$ & & \\
\hline
\end{tabular}

tions. Tetrahydrofuran (THF) was obtained from Merck (Darmstadt, Germany), and all other chemicals were ordered from Acros Organics (New Jersey, USA). THF was distilled from $\mathrm{Na}$ /benzophenone under $\mathrm{N}_{2}$. Diethylamine $\left(\mathrm{Et}_{2} \mathrm{NH}\right)$ was dried over $\mathrm{KOH}$ pellets and distilled under vacuum. All NMR solvents were purchased from Cambridge Isotope Lab., Inc. (MA, USA) and were used as received. $\operatorname{Pd}\left(\mathrm{PPh}_{3}\right)_{4}$ was prepared according to the literature [17]. Chromatographic purification was performed with Silica Gel 60 (230-400 mesh, Merck).

\subsection{Synthesis}

One hundred milligram of bromonated NiBPP under nitrogen was mixed with 2-4 equivalents of 2-pyridylethynye ( 2 eq. for reacting with $\mathrm{NiBPPBr}$ and 4 eq. for reacting with $\mathrm{NiBPPBr} 2), 20 \mathrm{~mol} \%$ of $\mathrm{Pd}\left(\mathrm{PPh}_{3}\right)_{4}$, $10 \mathrm{~mol} \%$ of $\mathrm{CuI}, 5 \mathrm{ml}$ of $\mathrm{Et}_{2} \mathrm{NH}$ and $80 \mathrm{ml}$ of THF.
The de-gassed reaction mixtures were stirred under nitrogen at $40{ }^{\circ} \mathrm{C}$ and monitored with TLC and UVVis spectroscopy. $\mathrm{NH}_{4} \mathrm{Cl}_{(\mathrm{aq})}$ washes were applied to quench the reactions. Porphyrin products were obtained after separation by chromatography on silica gel then crystallization from $\mathrm{CH}_{2} \mathrm{Cl}_{2} /$ hexanes. The reaction yields are listed in Table 1.

Other characterization data:

Ni 1': ${ }^{1} \mathrm{H}$ NMR $\left(\mathrm{CDCl}_{3}\right.$ at $\left.7.26 \mathrm{ppm}\right)$, porphine: 9.75(s, 1H) $9.31(\mathrm{~d}, J=5 \mathrm{~Hz}, 2 \mathrm{H}), 9.08(\mathrm{~d}, J=5 \mathrm{~Hz}$, $2 \mathrm{H}), 8.83(\mathrm{~d}, J=5 \mathrm{~Hz}, 2 \mathrm{H}), 8.81(\mathrm{~d}, J=5 \mathrm{~Hz}, 2 \mathrm{H})$, 8.01(m, 4H), 7.70(m, 6H); 2-pyridine-acetyl: 8.93(d, $J=5 \mathrm{~Hz}, 1 \mathrm{H}), 8.14(\mathrm{~d}, J=7 \mathrm{~Hz}, 1 \mathrm{H}), 7.90(\mathrm{t}, J=7 \mathrm{~Hz}$, $1 \mathrm{H}), 7.60(\mathrm{t}, J=6 \mathrm{~Hz}, 1 \mathrm{H}), 6.62(\mathrm{~s}, 2 \mathrm{H})$. Elemental analysis, $\mathrm{C}_{39} \mathrm{H}_{25} \mathrm{~N}_{5} \mathrm{ONi} \cdot \frac{1}{2} \mathrm{H}_{2} \mathrm{O}$, calculated $\mathrm{C} 72.36 \%, \mathrm{H} 4.05 \%$, N $10.82 \%$; Found C $72.70 \%$, H $3.99 \%$, N 10.55\%.

Ni 1 ${ }^{1} \mathrm{H}$ NMR $\left(\mathrm{CDCl}_{3}\right.$ at $\left.7.26 \mathrm{ppm}\right)$, porphine: 9.73 (s, $1 \mathrm{H}), 9.69(\mathrm{~d}, J=5 \mathrm{~Hz}, 2 \mathrm{H}), 9.03(\mathrm{~d}, J=5 \mathrm{~Hz}, 2 \mathrm{H}), 8.84(\mathrm{~d}$, $J=5 \mathrm{~Hz}, 2 \mathrm{H}), 8.79(\mathrm{~d}, J=5 \mathrm{~Hz}, 2 \mathrm{H}), 8.01(\mathrm{~m}, 4 \mathrm{H})$, 7.71(m, 6H); 2-pyridine-ethynyl: 8.82(overlapped, $1 \mathrm{H})$, 
$7.95(\mathrm{~d}, J=8 \mathrm{~Hz}, 1 \mathrm{H}), 7.87(\mathrm{t}, J=8 \mathrm{~Hz}, 1 \mathrm{H}), 7.39(\mathrm{t}, J=6$ $\mathrm{Hz}, 1 \mathrm{H})$. Elemental analysis, $\mathrm{C}_{39} \mathrm{H}_{23} \mathrm{~N}_{5} \mathrm{Ni} \cdot \mathrm{H}_{2} \mathrm{O}$, calculated C $73.38 \%$, H 3.95\%, N 10.97\%; Found C 73.56\%, H $3.79 \%$, N $10.36 \%$.

$\mathrm{Ni} \underline{2}^{\prime}:{ }^{1} \mathrm{H}$ NMR $\left(\mathrm{CDCl}_{3}\right.$ at $\left.7.26 \mathrm{ppm}\right)$, porphine: 9.59(d, $J=5 \mathrm{~Hz}, 2 \mathrm{H}), 9.21(\mathrm{~d}, J=5 \mathrm{~Hz}, 2 \mathrm{H}), 8.76(\mathrm{~d}$, $J=5 \mathrm{~Hz}, 2 \mathrm{H}), 8.70(\mathrm{~d}, J=5 \mathrm{~Hz}, 2 \mathrm{H}), 7.97(\mathrm{~m}, 4 \mathrm{H})$, 7.68(m, 6H); 2-pyridine-acetyl: $8.91(\mathrm{~d}, J=5 \mathrm{~Hz}, 1 \mathrm{H})$, $8.14(\mathrm{t}, J=7 \mathrm{~Hz}, 1 \mathrm{H}), 7.85(\mathrm{t}, J=7 \mathrm{~Hz}, 1 \mathrm{H}), 7.60(\mathrm{t}$, $J=6 \mathrm{~Hz}, 1 \mathrm{H}), 6.51(\mathrm{~s}, 2 \mathrm{H}) ; 2$-pyridine-ethynyl: $8.91(\mathrm{~d}$, $J=5 \mathrm{~Hz}, 1 \mathrm{H}), 7.92(\mathrm{~d}, J=8 \mathrm{~Hz}, 1 \mathrm{H}), 7.68$ (overlapped, $1 \mathrm{H}), \quad 7.37(\mathrm{t}, \quad J=6 \mathrm{~Hz}, 1 \mathrm{H}) . \quad$ Elemental analysis, $\mathrm{C}_{46} \mathrm{H}_{28} \mathrm{~N}_{6} \mathrm{ONi} \cdot \frac{1}{2} \mathrm{H}_{2} \mathrm{O}$, calculated C $73.82 \%$, H $3.91 \%$, N $11.23 \%$; Found C $73.95 \%$, H 3.88\%, N 11.22\%.

\section{Supplementary material}

Crystallographic data for the structural analysis of $\mathrm{Ni}$ $\underline{\mathbf{1}}^{\prime}$ has been deposited with the Cambridge Crystallographic Data Centre, CCDC No. 221509. Copies of this information may be obtained free of charge from: The Director, CCDC, 12 Union Road, Cambridge, CB2 1EZ UK (fax: +44 1223336 033, e-mail: deposit@ccdc. cam.ac.uk or www:http://www.ccdc.cam.ac.uk).

\section{Acknowledgements}

This work is supported by the National Science Council (NSC 92-2113-M-260-008). We also thank Professor Long-Li Lai and Professor Chen-Yu Yeh for the useful discussions.

\section{References}

[1] (a) N. Endoh, Y. Yonezawa, C.-G. Shin, Bull. Chem. Soc. Jpn. 76 (2003) 643-644;

(b) J.F. Callahan, J.L. Burgess, J.A. Fornwald, L.M. Gaster, J.D. Harling, F.P. Harrington, J. Heer, C. Kwon, R. Lehr, A. Mathur, B.A. Olson, J. Weinstock, N.J. Laping, J. Med. Chem. 45 (2002) 999-1001;

(c) A. Kolocouris, K. Dimas, C. Pannecouque, M. Witvrouw, G.B. Foscolos, G. Stamatiou, G. Fytas, G. Zoidis, N. Kolocouris, G. Andrei, R. Snoeckc, E. De Clercq, Bioorg. Med. Chem. Lett. 12 (2002) 723-727;

(d) B. Neelam, M. Mannar R., N. Fehmida, B. Alok, B. Sudha, A. Amir, Eur. J. Med. Chem. 35 (2000) 481-486;

(e) M. Journet, D. Cai, R.D. Larsen, P.J. Reider, Tetrahedron Lett. 39 (1998) 1717-1720;

(f) D.L. Klayman, A.J. Lin, J.W. McCall, S.-Y. Wang, S. Townson, M. Grogl, K.E. Kinnamon, J. Med. Chem. 34 (1991) 1422-1425.

[2] (a) R. Kramer, I.O. Fritsky, H. Pritzkow, L.A. Kovbasyuk, J. Chem. Soc., Dalton Trans. (2002) 1307-1314;

(b) M.A.S. Goher, F.A. Mautner, M.A.M. Abu-Youssef, A.K. Hafez, A.M.-A. Badr, J. Chem. Soc., Dalton Trans. (2002) 3309 3312 ;

(c) G.W.V. Cave, C.L. Raston, J. Chem. Soc., Perkin Trans. 1 (2001) 3258-3264; (d) J.-P. Sauvage, J.-P. Collin, J.-C. Chambron, S. Guillerez, C. Coudret, V. Baltani, F. Barigelletti, L. De Cola, L. Flamigni, Chem. Rev. 94 (1994) 993-1019.

[3] (a) D. Kovala-Demertzi, M.A. Demertzis, J.R. Miller, C. Papadopoulou, C. Dodorou, G. Filousis, J. Inorg. Biochem. 86 (2001) 555-563;

(b) Z. Iakovidou, A. Papageorgiou, M.A. Demertzis, E. Mioglou, D. Mourelatos, A. Kotsis, P.N. Yadav, D. Kovala-Demertzi, Anti-Cancer Drug 12 (2001) 65-70;

(c) V.B. Arion, M.A. Jakupec, M. Galanski, P. Unfried, B.K. Keppler, J. Inorg. Biochem. 91 (2002) 298-305.

[4] G. Chelucci, R.P. Thummel, Chem. Rev. 102 (2002) 3129-3170.

[5] (a) S. Chowdhury, P.B. Iveson, M.G.B. Drew, D.A. Tocher, D. Datta, New J. Chem. 27 (2003) 193-196;

(b) V. Berl, I. Huc, R.G. Khoury, M.J. Kirsche, J.-M. Lehn, Nature 407 (2000) 720.

[6] For recent review: K.M. Kadish, K.M. Smith, G. Guilard (Eds.), The Porphyrin Handbook, vol. 6, Academic Press, New York, 2000.

[7] (a) F.H. Case, W.A. Butte, J. Org. Chem. 26 (1961) 4415-4418; (b) H.R. Henze, M.B. Knowles, J. Org. Chem. 19 (1954) 1127 1135 ;

(c) F.B. LaForge, J. Am. Chem. Soc. 50 (1928) 2477-2483.

[8] (a) N. Miyaura, T. Yanagi, A. Suzuki, Synthetic Commun. 11 (1981) 513-519;

(b) A.G. Hyslop, M.A. Kellett, P.M. Iovine, M.J. Therien, J. Am. Chem. Soc. 120 (1998) 12676-12677.

[9] L.D. Ciana, A.J. Haim, Heterocyclic Chem. 21 (1984) 607-608.

[10] (a) K. Sonogashira, Y. Tohda, N. Hagihara, Tetrahedron Lett. (1975) 4467-4470;

(b) S. Takahashi, Y. Kuroyama, K. Sonogashira, Synthesis (1980) 627-630.

[11] (a) S.M. LeCours, C.M. Philips, J.C. de Paula, M.J. Therien, J. Am. Chem. Soc. 119 (1997) 12578-12589;

(b) S.M. LeCours, H.W. Guan, S.G. DiMagno, C.H. Wang, M.J. Therien, J. Am. Chem. Soc. 118 (1996) 1497-1503.

[12] (a) F. Li, S.I. Yang, Y. Ciringh, J. Seth, C.H. Martin III, D.L. Singh, D. Kim, R.R. Birge, D.F. Bocian, D. Holten, J.S. Lindsey, J. Am. Chem. Soc. 120 (1998) 10001-10017;

(b) N. Nishino, R.W. Wagner, J.S. Lindsey, J. Org. Chem. 61 (1996) 7534-7544.

[13] (a) I.M. Blake, L.H. Rees, T.D.W. Claridge, H.L. Anderson, Angew. Chem. Int. Ed. 39 (2000) 1818-1821;

(b) P.N. Taylor, A.P. Wylie, J. Huuskonen, H.L. Anderson, Angew. Chem. Int. Ed. 37 (1998) 986-989.

[14] S.L. Darling, C.C. Mak, N. Bampos, N. Feeder, S.J. Teat, J.K.M. Sanders, New J. Chem. 23 (1999) 359-364.

[15] (a) D.P. Arnold, D.A. James, J. Org. Chem. 62 (1997) 3460-3469; (b) R. Stranger, J.E. McGrady, D.P. Arnold, I. Lane, G.A. Heath, Inorg. Chem. 35 (1996) 7781-7797.

[16] T. Sakamoto, Y. Kondo, M. Shiraiwa, H. Yamanaka, Synthesis 3 (1984) 245-247.

[17] H.M. Colquhoun, J. Holton, D.J. Thompson, W.V. Twigg (Eds.), New pathways for Organic Synthesis: Pratial Applications of Transition Metals, Plenum Press, New York, 1984, pp. 383384.

[18] C.-Y. Lin, L.-C. Chuang, Y.-F. Yang, C.-L. Lin, H.-C. Kao, W.J. Wang, Dalton Trans. (2004) 456-462.

[19] (a) Examples of zinc porphyrins with pyridine or THF axial ligands (crystal structures): A.G. Hyslop, M.A. Kellett, P.M. Iovine, M.J. Therien, J. Am. Chem. Soc. 120 (1998) 12676-12677; (b) S.M. LeCours, S.G. DiMagno, M.J. Therien, J. Am. Chem. Soc. 118 (1996) 11854-11864.

[20] (a) S.J. Cole, G.C. Curthoys, E.A. Magnusson, J.N. Phillips, Inorg. Chem. 11 (1972) 1024-1028;

(b) D. Kim, Y.O. Su, T.G. Spiro, Inorg. Chem. 25 (1986) 39883993 (references therein). 